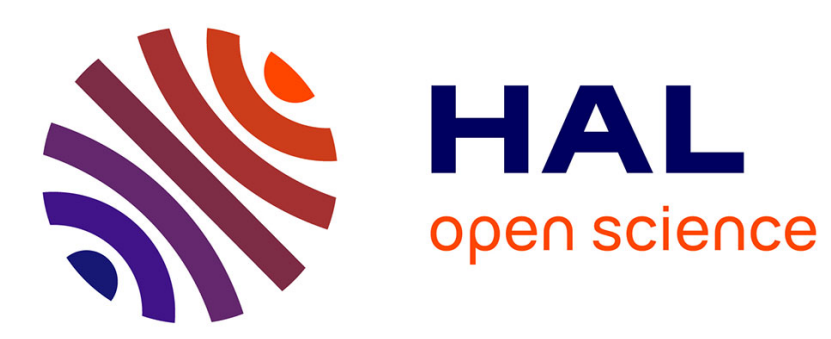

\title{
Post-Impact Adaptive Compliance for Humanoid Falls Using Predictive Control of a Reduced Model
}

Vincent Samy, Stéphane Caron, Karim Bouyarmane, Abderrahmane Kheddar

\section{To cite this version:}

Vincent Samy, Stéphane Caron, Karim Bouyarmane, Abderrahmane Kheddar. Post-Impact Adaptive Compliance for Humanoid Falls Using Predictive Control of a Reduced Model. Humanoids, Nov 2017, Birmingham, United Kingdom. pp.655-660, 10.1109/HUMANOIDS.2017.8246942 . hal-01569819v3

\section{HAL Id: hal-01569819 \\ https://hal.science/hal-01569819v3}

Submitted on 2 Nov 2017

HAL is a multi-disciplinary open access archive for the deposit and dissemination of scientific research documents, whether they are published or not. The documents may come from teaching and research institutions in France or abroad, or from public or private research centers.
L'archive ouverte pluridisciplinaire HAL, est destinée au dépôt et à la diffusion de documents scientifiques de niveau recherche, publiés ou non, émanant des établissements d'enseignement et de recherche français ou étrangers, des laboratoires publics ou privés. 


\title{
Post-Impact Adaptive Compliance for Humanoid Falls Using Predictive Control of a Reduced Model
}

\author{
Vincent Samy ${ }^{1}$, Stéphane Caron ${ }^{1}$, Karim Bouyarmane ${ }^{2}$ and Abderrahmane Kheddar ${ }^{1,3}$
}

\begin{abstract}
We consider control of a humanoid robot in active compliance just after the impact consecutive to a fall. The goal of this post-impact braking is to absorb undesired linear momentum accumulated during the fall, using a limited supply of time and actuation power. The gist of our method is an optimal distribution of undesired momentum between the robot's hand and foot contact points, followed by the parallel resolution of Linear Model Predictive Control (LMPC) at each contact. This distribution is made possible thanks to torquelimited friction polytopes, an extension of friction cones that takes actuation limits into account. Individual LMPC results are finally combined back into a feasible CoM trajectory sent to the robot's whole-body controller. We validate the solution in full-body dynamics simulation of an HRP-4 humanoid falling on a wall.
\end{abstract}

\section{INTRODUCTION}

Designing humanoids robots with robust falling and recovery strategies is as important as agile walking or whole-body attitude control. The DARPA Robotics Challenge highlighted how falls that are not properly handled result in substantial hardware damage. Several fall avoidance strategies were thus experimented, including reliable but conservative approaches [1], foothold replacement on the fly [2], planning of additional contacts on the fly [3], etc. There is certainly room for improvement, and such strategies shall be further investigated. Nevertheless, even the most sophisticated legged creatures in nature do end-up in situations were fall is inevitable, and so will humanoid robots. Our research is focused on dealing with falls when they occur and that cannot be recovered from by trying to reduce damage as much as possible.

A fall incurs relatively high impacts on the mechanical structure of the robot, including linkage and actuators. Unfortunately, there is not much to do at the software level to deal with the propagation of mechanical impacts. Our conviction is that the hardware design of humanoid robots must embed impact-absorbing components at any rate, e.g. flexible covers [4], compliant contacting interfaces [5] or compliant joint actuators coupled with gains tuning algorithms [6]. They can be complemented by active or semi-active methods to reduce impact intensity (pre-impact prevention) or further absorb its effect (post-impact compliance).

In our previous work [7], we leveraged the brief amount of time available in the pre-impact landing phase to adapt the humanoid's posture based on a predefined taxonomy of postures suited to each fall direction. Then, to deal with the post-impact phase, we suggested reducing $\mathrm{PD}$ gains in the

\footnotetext{
1 IDH group, CNRS-UM LIRMM, UMR5506, Montpellier, France.

2 Université de Lorraine-INRIA-CNRS LORIA, 54600 Villers-lès-Nancy, France.

3 CNRS-AIST Joint Robotics Laboratory (JRL), UMI3218/RL, Japan.

* This work is supported in part by H2020 EU project COMANOID http://www. comanoid.eu/ RIA No 645097 and ROMEO2 project www.projetromeo.com

Corresponding author: vincent. samyalirmm. fr
}

low-level actuator controllers to realize a compliant springdamper-like behavior at the joint level. These post-impact PD gains, first determined ad hoc from experiments, were later derived automatically using a gain-adaptive whole-body quadratic-programming controller [8]. We call this control scheme "adaptive QP". An adaptive-QP controller enables the robot to comply during post-impact with $\mathrm{PD}$ gains computed in accordance with its state variables. Combining posture selection and gain adaption, we carried out real-world experiments where the HRP-4 humanoid robot sustained front and back falls on a soft mattress, starting from an upright standing posture.

Post-impact recovery is about absorbing undesired momentum, accumulated during the fall, using contact forces. This momentum cannot be absorbed instantly due to friction and actuation limits. However, it must be absorbed before position limits (joint limits or collision with the environment) are reached. The adaptive-QP scheme, being an instantaneous and reactive control scheme, lacks the level of foresight necessary to reach an efficient balance between these two kinds of limitations.

The contribution of the present work is to complement adaptive-QP whole-body control with an adaptivecompliance predictive controller that satisfies both postimpact actuation and position limits. Due to the size of receding-horizon problems, predictive control requires the use of reduced dynamic models, which usually cannot represent whole-body actuation limits. To overcome this, we introduce a novel mapping of joint-torque limits into actuation polytopes that generalize friction cones. We then distribute momentum between contacts and solve the predictive control problem using parallel computations, resulting in a reduced-model trajectory that is finally tracked by adaptive$\mathrm{QP}$ control. The validity of this approach is demonstrated in simulations with the HRP-4 humanoid robot.

\section{BACKGROUND}

\section{A. Gain-adaptive QP-based whole-body control}

In our recent work [8], we extended our whole-body QP task-space controller [9] to include PD gains of the actuators as part of the QP decision variables. We also demonstrated that such a scheme applies to position or torque controlled robots. The reason behind this approach was to automate PDgains tuning so as to make the robot compliant during the post-impact phase, and hence reduce damage by absorbing as much as possible the momentum accumulated while falling. The PD coefficients have been inserted into the decision 
vector of a QP whole-body control problem as follows:

$$
\begin{aligned}
\min _{\ddot{\boldsymbol{q}}, \boldsymbol{\lambda}, \boldsymbol{K}, \boldsymbol{B}} & \sum_{k} \omega_{k}^{\mathrm{sp}} E_{k}^{\mathrm{sp}}+\omega_{\lambda}\|\boldsymbol{\lambda}\|^{2}+\omega_{G}\left(\|\boldsymbol{K}\|^{2}+\|\boldsymbol{B}\|^{2}\right), \\
\text { s.t. } \quad & H_{S} \ddot{\boldsymbol{q}}+\boldsymbol{c}_{S}-\left(J^{T} G\right)_{S} \boldsymbol{\lambda}=K \boldsymbol{e}_{S}-B \dot{\boldsymbol{e}}_{S} \\
& \underline{\boldsymbol{\tau}}_{S} \leq K \boldsymbol{e}_{S}-B \dot{\boldsymbol{e}}_{S} \leq \overline{\boldsymbol{\tau}}_{S} \\
& \underline{\boldsymbol{\tau}}_{\mathrm{NS}} \leq H_{\mathrm{NS}} \ddot{\boldsymbol{q}}+\boldsymbol{c}_{\mathrm{NS}}-\left(J^{T} G\right)_{\mathrm{NS}} \boldsymbol{\lambda} \leq \overline{\boldsymbol{\tau}}_{\mathrm{NS}} \\
& \underline{\ddot{\boldsymbol{q}}} \leq \ddot{\boldsymbol{q}} \leq \overline{\boldsymbol{q}}, \boldsymbol{\lambda} \geq \mathbf{0}, \boldsymbol{K} \geq \mathbf{0}, \boldsymbol{B} \geq \mathbf{0}
\end{aligned}
$$

with $\ddot{q} \in \mathbb{R}^{N}$ the vector of generalized joint accelerations, $\lambda \in \mathbb{R}^{N_{G}}$ the generator weights of linearized friction cones, $\boldsymbol{K} \in \mathbb{R}^{N-6}$ and $\boldsymbol{B} \in \mathbb{R}^{N-6}$ the vectors of stiffness and damping coefficients vector, $\omega_{k}$ the positive weight of task $k, E_{k}^{\mathrm{sp}}$ the residual (error) vector of task $k, H \in \mathbb{R}^{N \times N}$ the inertia matrix, $c \in \mathbb{R}^{N}$ the vector of Coriolis and gravity forces, $J^{T} G \in \mathbb{R}^{N \times N_{G}}$ the mapping from linearized friction cones to the joint space and $\boldsymbol{e}=\boldsymbol{q}^{\text {ref }}-\boldsymbol{q}$ the vector of joint deviations from a reference posture $q^{\text {ref }}$. The subscript $S$ denotes joints that are set to be compliant, for which non-zero entries will appear in the vectors $\boldsymbol{K}$ and $\boldsymbol{B}$. The subscript $N S$ denotes joint that are not set to be compliant. Generally, $\boldsymbol{q}^{\text {ref }}$ is set as the state at the impact time, while $\dot{\boldsymbol{q}}^{\text {ref }}$ is zero. Finally, variables such as $\bar{\tau}$ (resp. $\underline{\tau}$ ) denote the upper bound (resp. lower bound) of the corresponding variables. The lower and upper bound for the free-floating base part of the dynamics equation is zero.

\section{B. Polyhedra and polytopes}

Polyhedra are convex sets realized by the intersection of finitely-many halfspaces. They correspond to the inequality constraints found in linear and quadratic programs, as well as the dual constraints such as friction cones or ZMP support areas that arise in mechanics [10]. A polytope is a bounded polyhedron. It can be represented equivalently as: $(i)$ the intersection of finitely-many halfspaces, called the $\mathcal{H}$-representation, or (ii) the convex hull of a finite set of vertices, called the $\mathcal{V}$-representation. The latter can be written mathematically as:

$$
\mathcal{P}=\left\{\boldsymbol{x}=\sum_{i=1}^{N} \alpha_{i} \boldsymbol{v}_{i} \mid \forall i, \alpha_{i} \geq 0, \sum_{i=1}^{N} \alpha_{i}=1\right\}
$$

where the positive coefficients $\alpha_{i}$ form a convex combination of the $N$ vertices $\boldsymbol{v}_{i}$ of the polytope. Meanwhile, the $\mathcal{H}$ representation can be concisely written as:

$$
A \boldsymbol{x} \leq \boldsymbol{b}
$$

where the matrix $A=\left[\boldsymbol{A}_{0} \ldots \boldsymbol{A}_{N}\right]^{T}$ stacks halfspace normals $\boldsymbol{A}_{i}$, so that $\boldsymbol{A}_{i}^{T} \boldsymbol{x}=b_{i}$ is the equation of the $i^{\text {th }}$ supporting halfspace of the polytope. The conversion from $\mathcal{H}$ to $\mathcal{V}$ (resp. from $\mathcal{V}$ to $\mathcal{H}$ ) representation of a polytope is known as the vertex enumeration problem. Both can be realized by the double-description algorithm [11].

\section{ReduCED DyNAMiC MODEL}

We approximate the whole-body dynamics at the center of mass (CoM) of the robot and search for contact forces that compensate for both gravity and post-impact linear momentum. The Newton equation of motion that governs the acceleration of the CoM is:

$$
\boldsymbol{F}=M(\ddot{\boldsymbol{s}}-\boldsymbol{g})
$$

with $M$ the total robot mass, $\ddot{s}$ the acceleration of the CoM, $\boldsymbol{g}$ the gravity vector and $\boldsymbol{F}$ the net contact force (sum of all external forces) applied to the robot.

Under the assumption that the initial linear momentum $M \dot{\boldsymbol{s}}^{0}$ at impact is known, the goal of post-impact absorption is to find a trajectory $\ddot{\boldsymbol{s}}(t)$ such that, at time $t=t_{f}, \dot{\boldsymbol{s}}\left(t_{f}\right)=$ 0 , meaning that all the linear momentum accumulated while falling has been driven out of the system. Achieving this task requires proper control of the net contact force $\boldsymbol{F}$.

\section{A. Actuation constraints}

Let us consider again the full-body dynamics equation of motion of the system

$$
H \ddot{\boldsymbol{q}}+\boldsymbol{c}=J^{T} \boldsymbol{F}+S^{T} \boldsymbol{\tau} .
$$

$\boldsymbol{F}=\left(J^{T} G\right) \boldsymbol{\lambda}$ is the stacked vector of contact forces and $S$ is a selection matrix of actuated joints among all the degrees of freedom (DoFs) of the robot (hence the matrix that excludes the floating-base DoFs from the total DoFs [12].

A fundamental property of the humanoid kinematic tree topology is that it comprises at least 4 limbs (two legs and two arms), indexed here with the variable $c \in\{\mathrm{lh}, \mathrm{rh}, \mathrm{lf}, \mathrm{rf}\}$, that extend from the root of the kinematic tree to the endeffectors (respectively the two hands and two feet). The contact force applied at the end-effector $c$ is denoted $\boldsymbol{F}_{c}$ (therefore we have $\boldsymbol{F}=\left(\boldsymbol{F}_{c}\right)_{c \in\{\mathrm{lh}, \mathrm{rh}, \mathrm{lf}, \mathrm{rf}\}}$ ). The rest of the DoFs, including the floating base are indexed with 0. Eq. (5) can be rewritten as

$$
\left[\begin{array}{c}
H_{0} \\
H_{\mathrm{lh}} \\
H_{\mathrm{rh}} \\
H_{\mathrm{lf}} \\
H_{\mathrm{rf}}
\end{array}\right] \ddot{\boldsymbol{q}}+\left[\begin{array}{c}
\boldsymbol{c}_{0} \\
\boldsymbol{c}_{\mathrm{lh}} \\
\boldsymbol{c}_{\mathrm{rh}} \\
\boldsymbol{c}_{\mathrm{lf}} \\
\boldsymbol{c}_{\mathrm{rf}}
\end{array}\right]=\left[\begin{array}{cccc}
J_{0, \mathrm{hh}}^{T} & J_{0, \mathrm{rh}}^{T} & J_{0, \mathrm{lf}}^{T} & J_{0, \mathrm{rf}}^{T} \\
J_{\mathrm{lh}}^{T} & 0 & 0 & 0 \\
0 & J_{\mathrm{rh}}^{T} & 0 & 0 \\
0 & 0 & J_{\mathrm{lf}}^{T} & 0 \\
0 & 0 & 0 & J_{\mathrm{rf}}^{T}
\end{array}\right]\left[\begin{array}{c}
\boldsymbol{F}_{\mathrm{lh}} \\
\boldsymbol{F}_{\mathrm{rh}} \\
\boldsymbol{F}_{\mathrm{lf}} \\
\boldsymbol{F}_{\mathrm{rf}}
\end{array}\right]+\left[\begin{array}{c}
\boldsymbol{\tau}_{0} \\
\boldsymbol{\tau}_{\mathrm{lh}} \\
\boldsymbol{\tau}_{\mathrm{rh}} \\
\boldsymbol{\tau}_{\mathrm{lf}} \\
\boldsymbol{\tau}_{\mathrm{rf}}
\end{array}\right],
$$

where $H_{c}, \boldsymbol{c}_{c}, \boldsymbol{\tau}_{c}$ are the rows of respectively $H, \boldsymbol{c}$ and $\boldsymbol{\tau}$ and $J_{c}$ is the reduced Jacobian of the contact points at the end-effector $c$ to the root of the kinematic tree, with respect only to the set of joints of the considered limb (and not with respect to all the DoFs of the robot). The contact force at limb $c \in\{$ lh, rh, lf, rf $\}$. $\boldsymbol{F}_{c}$, only affects the part of the dynamics equation in $\{c, 0\}$ :

$$
H_{c} \ddot{\boldsymbol{q}}+\boldsymbol{c}_{c}=J_{c}^{T} \boldsymbol{F}_{c}+\boldsymbol{\tau}_{c} .
$$

Using the reduced Jacobian, the kinematic contact constraint at the end-effector $c$ is expressed in acceleration-form as:

$$
J_{c} \ddot{\boldsymbol{q}}+\dot{J}_{c} \dot{\boldsymbol{q}}=\mathbf{0} .
$$

Substituting Eq. (7) in Eq. (8) yields

$$
\begin{aligned}
J_{c} H_{c}^{-1} J_{c}^{T} \boldsymbol{F}_{c} & =J_{c} H_{c}^{-1}\left(\boldsymbol{c}_{c}-\boldsymbol{\tau}_{c}\right)-\dot{J}_{c} \dot{\boldsymbol{q}} \\
\Lambda_{c}^{-1} \boldsymbol{F}_{c} & =-J_{c} H_{c}^{-1} \boldsymbol{\tau}_{c}+J_{c} H_{c}^{-1} \boldsymbol{c}-\dot{J}_{c} \dot{\boldsymbol{q}} \\
\boldsymbol{F}_{c} & =-\Lambda_{c} A_{c} \boldsymbol{\tau}_{c}+\boldsymbol{d}_{c} \\
\boldsymbol{F}_{c} & =C_{c} \boldsymbol{\tau}_{c}+\boldsymbol{d}_{c} .
\end{aligned}
$$

The matrix $\Lambda_{c}$ is the operational space inertia matrix at point $c, C_{c}$ and $\boldsymbol{d}_{c}$ provide an affine mapping from torque limits to contact force limits. The $\mathcal{H}$-representation of the torqueconstraint polytope is:

$$
\underline{\boldsymbol{\tau}}_{c} \leq \boldsymbol{\tau}_{c} \leq \overline{\boldsymbol{\tau}}_{c}
$$




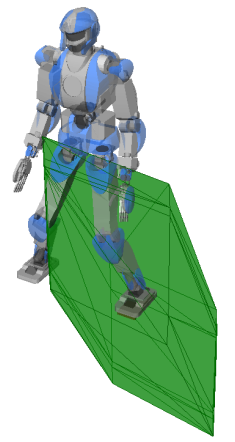

(a)

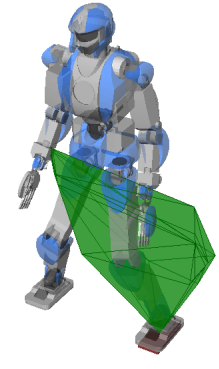

(b)
Fig. 1: Torque-limited friction polytopes. Feasible contact forces at the left foot of HRP-4 under (a) leg torque limits and (b) both torque limits and friction constraints.

where $\tau_{c}$ is the vector of selected joint torques for the contact $c$ (corresponding to the limb at hand, e.g. left-leg joints for a left-foot contact), $\underline{\tau}_{c}$ and $\bar{\tau}_{c}$ are respectively the lower and upper torque limits of the actuators. Applying Eq. (9) yields the $\mathcal{V}$-representation of the torque-limited polytope of feasible end-effector forces at the contact point, depicted in Fig 1a Using the double-description method, we convert it to $\mathcal{H}$-representation:

$$
G_{c}^{\text {actuators }} \boldsymbol{F}_{c} \leq \boldsymbol{h}_{c}^{\text {actuators }} .
$$

\section{B. Friction constraints}

The next actuation limit at contact comes from friction. Using a linearized friction pyramid, whose $\mathcal{H}$-representation is given by (see e.g. [10]):

$$
\left[\begin{array}{ccc}
-1 & 0 & -\mu \\
1 & 0 & -\mu \\
0 & -1 & -\mu \\
0 & 1 & -\mu
\end{array}\right]{ }^{0} E_{c} \boldsymbol{F}_{c} \leq \mathbf{0}
$$

where $\mu$ is the friction coefficient and ${ }^{0} E_{c}$ is the rotation matrix from the contact frame to the world frame.

Concatenating Eq. (11) and Eq. (12) leads to the $\mathcal{H}$ representation

$$
G_{c} \boldsymbol{F}_{c} \leq \boldsymbol{h}_{c},
$$

of the torque- and friction-constrained actuation polytope depicted in Fig. $1 \mathrm{~b}$

\section{DISTRIBUTION OF GRAVITY AND LINEAR MOMENTUM}

Our primary idea is to make each limb $c \in\{$ lh, rh, lf, rf $\}$ of the robot contribute, in post-impact, to a "share" of the pre-impact linear momentum absorption, summing up all contributions into the resultant equation of motion:

$$
\sum_{c \in\{\mathrm{lh}, \mathrm{rh}, \mathrm{lf}, \mathrm{rf}\}} \boldsymbol{F}_{c}=M(\ddot{\boldsymbol{s}}-\boldsymbol{g}) .
$$

This can be conceptually interpreted as "splitting" the CoM into four virtual mass points with state vectors $\left(\boldsymbol{s}_{c}, \dot{\boldsymbol{s}}_{c}\right)$, each going towards one of the limb extremities in contact, and each with a force $\boldsymbol{F}_{c}$ applied to it. Hence we are solving for the system of four differential equations for the state variables $\left(\boldsymbol{s}_{c}, \dot{\boldsymbol{s}}_{c}\right)$ :

$$
\boldsymbol{F}_{c}=M\left(\ddot{\boldsymbol{s}}_{c}-\boldsymbol{g}_{c}\right)
$$

where $\boldsymbol{g}_{c}$ is a quantity to be defined below (gravity force distribution problem) and where the initial condition for each component is also to be defined below (initial impact momentum distribution problem). The force $\boldsymbol{F}_{c}$ is used to decelerate its respective CoM component until full initial momentum absorption, while being contrained to lie inside its respective actuation limit polytope (Section III-A).

Therefore, in the following, we distribute the amount of gravity and initial linear momentum that each contact will support. This distribution must satisfy (Eq. (14)). we also want to make sure that the distribution is optimal with respect to the actuation constraint polytope at each contact.

Assuming that the linear momentum $\boldsymbol{p}$ after impact is known, a corresponding force quantity $\boldsymbol{f}^{a}$ that would be physically consistent with the problem of distribution in the respective contact force polytopes is searched. The force being a time-derivative of the linear momentum, we have:

$$
\boldsymbol{f}=\frac{d \boldsymbol{p}}{d t}=M \frac{d \dot{\boldsymbol{s}}}{d t}
$$

In the worst case where the robot is completely rigid and the coefficient of restitution of the impact is zero, the impact force corresponding to the momentum $M \dot{\boldsymbol{s}}^{0}$ is:

$$
\boldsymbol{f}=\lim _{h \rightarrow 0} M \frac{\dot{\boldsymbol{s}}^{0}}{h}
$$

Because this form is non-linear in $h$, we set $k=1 / h$ (hence $k$ can be seen as a "gain" that maps a linear momentum quantity to a force quantity). The limit becomes

$$
\boldsymbol{f}=\lim _{k \rightarrow \infty} k M \dot{\boldsymbol{s}}^{0}
$$

Then we define $\boldsymbol{f}^{a}$ as the exact applied force

$$
\boldsymbol{f}^{a} \triangleq k M \dot{\boldsymbol{s}}^{0}
$$

where $k$ is a parameter that approach infinity. $\boldsymbol{f}^{a}$ corresponds to the average impact force for impact time $1 / k$.

Being already a force quantity, the gravity force:

$$
\boldsymbol{f}^{g}=M \boldsymbol{g}
$$

can be readily distributed in the polytopes.

We now need to find which contact will better handle $\boldsymbol{f}^{g}$ and which will better handle $f^{a}$ (as in Fig. 2 where the feet of the robot are more suitable for compensating the gravity than the hands). From Eq. (2) we split $\alpha_{i}$ in two variables $\alpha_{i}^{g}$ and $\alpha_{i}^{a}$ corresponding respectively to the percentage of $\boldsymbol{f}^{g}$ and $\boldsymbol{f}^{a}$ to address to the contact.

$$
\sum_{i} \alpha_{i}=\sum_{i} \alpha_{i}^{g}+\alpha_{i}^{a}
$$

This is done for each contact $c$. Using Eq. (2), (19), 20) and 


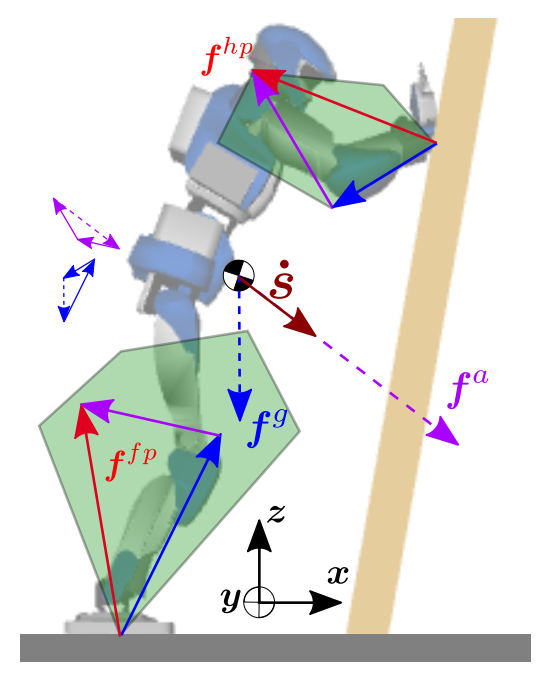

Fig. 2: Optimized contact force distribution. 2D illustration of a potential scenario. Dotted arrows $\boldsymbol{f}^{g}$ and $\boldsymbol{f}^{a}$ are forces to be distributed. Plain arrows show their repartition inside force polytopes: $f^{\mathrm{fp}}$ and $f^{\mathrm{hp}}$ have to remain inside their respective polytope. The dotted vector $f^{a}$ is taken so as to maximize the linear momentum coming from the CoM velocity $\dot{s}$.

(21) we can write the following constraints

$$
\begin{aligned}
\sum_{c} \sum_{i} \alpha_{c, i}^{g} \boldsymbol{v}_{c, i} & =-M \boldsymbol{g} \\
\sum_{c} \sum_{i} \alpha_{c, i}^{a} \boldsymbol{v}_{c, i} & =-k M \dot{\boldsymbol{s}}^{0} \\
\sum_{i} \alpha_{c, i}^{g}+\alpha_{c, i}^{a} & \leq 1 \\
\alpha_{c, i}^{g} & \geq 0 \\
\alpha_{c, i}^{a} & \geq 0 \\
k & \geq 0 .
\end{aligned}
$$

Constraints Eq. (22) and Eq. (23) distribute forces considering the polytope of each contact. Constraint Eq. (24) is a generalization of Eq. (2). Substituting the equality to an inequality does not change the definition of the polytope and relax the constraint. And finally, Eq. (25)-(27) make the variables consistent with the polytope definition (2) and the force generated at the CoM definition (19).

To have a good distribution over the feet and the hands (i.e. to avoid having the robot's weight supported by one foot), we design the following cost functions

$$
\left\{\begin{array}{c}
\left\|\sum_{i} \alpha_{\mathrm{rf}, i}^{g} \boldsymbol{v}_{\mathrm{rf}, i}-\sum_{i} \alpha_{\mathrm{lf}, i}^{g} \boldsymbol{v}_{\mathrm{lf}, i}\right\|^{2} \\
\left\|\sum_{i} \alpha_{\mathrm{rh}, i}^{a} \boldsymbol{v}_{\mathrm{rh}, i}-\sum_{i} \alpha_{\mathrm{lh}, i}^{a} \boldsymbol{v}_{\mathrm{lh}, i}\right\|^{2} \\
-k
\end{array}\right.
$$

As we want the variable $k$ to approach infinity, a cost function is set to maximize its value in the problem.

The overall system, with linear constraints Eq. (22)(27) and quadratic cost functions Eq. (28) is a Quadratic Programming (QP) problem. Later on, we call it the Force Distribution QP (FDQP). Fig. 2 shows a possible repartition of the forces over two contact points. The FDQP looks for a solution that compensates the force from gravity and maximizes the force generated against linear momentum.
Once the solution is found, $\alpha_{c}^{g}$, $\alpha_{c}^{a}$ and $k$ can be computed using the FDQP output:

$$
\begin{aligned}
-M \boldsymbol{g}_{c} & =\sum_{i} \alpha_{c, i}^{g} \boldsymbol{v}_{c, i}, \\
-k M \dot{\boldsymbol{s}}_{c}^{0} & =\sum_{i} \alpha_{c, i}^{a} \boldsymbol{v}_{c, i} .
\end{aligned}
$$

These two latter equations make Eq. (15) fully specified, with the specification of the constant vector $\boldsymbol{g}_{c}$ and the initial condition $\dot{\boldsymbol{s}}^{0}$.

\section{COM TRAJECTORY SOLUTION}

We solve the system Eq. (15) using a Linear Model Predictive Control (LMPC). Let $\boldsymbol{x}_{c}=\left[\begin{array}{ll}\boldsymbol{s}_{c}^{T} & \dot{\boldsymbol{s}}_{c}^{T}\end{array}\right]^{T}$ be the parameter vector. Eq. (15) can be put into matrix form:

$$
\begin{aligned}
\dot{\boldsymbol{x}}_{c} & =\left[\begin{array}{ll}
0_{3} & I_{3} \\
0_{3} & 0_{3}
\end{array}\right] \boldsymbol{x}_{c}+\left[\begin{array}{c}
0_{3} \\
1_{3} M^{-1}
\end{array}\right] \boldsymbol{u}+\left[\begin{array}{l}
\mathbf{0}_{3} \\
\boldsymbol{g}_{c}
\end{array}\right] \\
\dot{\boldsymbol{x}}_{c} & =\mathcal{A} \boldsymbol{x}_{c}+\mathcal{B} \boldsymbol{u}_{c}+\mathcal{E}_{c} .
\end{aligned}
$$

where $\dot{\boldsymbol{x}}_{c}=\left[\begin{array}{lll}\dot{\boldsymbol{s}}_{c}^{T} & \ddot{\boldsymbol{s}}_{c}^{T}\end{array}\right]^{T}$. This is a continuous time system with $\boldsymbol{u}=\boldsymbol{F}_{c}$, the control vector, $M$ the total mass of the system and $\boldsymbol{g}_{c}$ the contact distributed part of the gravity (computed with Eq. (29p). After discretization with a sampling time $T$ :

$$
\dot{\boldsymbol{x}}_{c}^{k+1}=\left[\begin{array}{cc}
I_{3} & I_{3} T \\
0_{3} & I_{3}
\end{array}\right] \dot{\boldsymbol{x}}_{c}^{k}+\left[\begin{array}{c}
I_{3} \frac{T^{2}}{2} \\
I_{3} T
\end{array}\right] \boldsymbol{u}^{k}+\left[\begin{array}{c}
\boldsymbol{g}_{c} \frac{T^{2}}{2} \\
\boldsymbol{g}_{c} T
\end{array}\right]
$$

The sampling time is not necessarily set to the robot control loop time. Indeed the greater the MPC's sampling time the wider is the horizon of time. Then an interpolation is done to get the values at each control loop time. By recursion we can compute a prediction of the behavior of the simplified system [13]. We only need to know the initialization parameter of the system.

After $N$ step, we get:

$$
\boldsymbol{X}_{c}^{N}=\Phi \boldsymbol{x}_{c}^{0}+\Psi \boldsymbol{U}_{c}+\boldsymbol{\zeta}_{c}
$$

with

$$
\begin{aligned}
\boldsymbol{X}_{c}^{N} & =\left[\begin{array}{llll}
\boldsymbol{x}_{c}^{0 T} & \boldsymbol{x}_{c}^{1 T} & \ldots & \boldsymbol{x}_{c}^{N T}
\end{array}\right]^{T} \\
\boldsymbol{U}_{c} & =\left[\begin{array}{lllll}
\boldsymbol{u}_{c}^{0 T} & \boldsymbol{u}_{c}^{1 T} & \ldots & \boldsymbol{u}_{c}^{N-1 T}
\end{array}\right]^{T}
\end{aligned}
$$

and where $\Phi, \Psi$ and $\boldsymbol{\zeta}_{c}$ are defined as in Eq. (35). Note that $\boldsymbol{x}_{c}^{0}=\left[\begin{array}{ll}\boldsymbol{s}^{0 T} & \dot{\boldsymbol{s}}_{c}^{0 T}\end{array}\right]^{T}$ where $\dot{\boldsymbol{s}}_{c}^{0}$ is the CoM distributed velocity (Eq. (30) ) and $s^{0}$ is the intial position of the CoM (which is the inital position of all four virtual CoMs).

The objective function of the LMPC is

$$
\begin{aligned}
t_{c}^{d} & =\left(\boldsymbol{x}_{c}^{N}-\boldsymbol{x}_{c}^{\mathrm{target}}\right) W_{x}\left(\boldsymbol{x}_{c}^{N}-\boldsymbol{x}_{c}^{\mathrm{target}}\right)+\boldsymbol{U}_{c}^{\boldsymbol{T}} W_{U} \boldsymbol{U}_{c} \\
& =\boldsymbol{U}_{c}^{T} Q_{c} \boldsymbol{U}_{c}+2 l_{c}^{T} \boldsymbol{U}_{c} .
\end{aligned}
$$

The target $\boldsymbol{x}_{c}^{\text {target }}=\left[\boldsymbol{s}_{c}^{\text {target T }} \boldsymbol{s}_{c}^{\text {target } T}\right]^{T}$ is such that $\boldsymbol{s}_{c}^{\text {target }}=\boldsymbol{s}_{c}^{0}$ and $\dot{\boldsymbol{s}}_{c}^{\text {target }}=\mathbf{0}$. The weights $W_{x}$ and $W_{U}$ are chosen so that the priority is to reach a zero velocity, then maintain the position and lastly minimize the effort. Briefly, this means that $W_{x}(3 . .6) \gg W_{x}(0 . .2)>W_{U}$. The force limit constraint (computed via Eq. (13)) is added to the system constraints. We then solve the LMPCs corresponding to each contact to get the vectors $\boldsymbol{U}_{c}$ and deduce the forces to apply at the contact points. As the adaptive-QP of the robot allows us to 


$$
\Phi=\left[\begin{array}{c}
A \\
A^{2} \\
\vdots \\
A^{N}
\end{array}\right], \quad \Psi=\left[\begin{array}{cccc}
B & 0 & \ldots & 0 \\
A B & B & \ddots & \vdots \\
\vdots & \vdots & \ddots & 0 \\
A^{N-1} B & A^{N-2} B & \ldots & B
\end{array}\right], \quad \boldsymbol{\zeta}_{c}=\left[\begin{array}{c}
\boldsymbol{E}_{c} \\
A \boldsymbol{E}_{c}+\boldsymbol{E}_{c} \\
\vdots \\
\sum_{i=0}^{i=N-1} A^{i} \boldsymbol{E}_{c}
\end{array}\right]
$$

set a trajectory task for the CoM, the forces are converted into CoM acceleration.

$$
\ddot{\boldsymbol{s}}(t)=\frac{1}{M} \sum_{c} \boldsymbol{F}_{c}(t)+\boldsymbol{g} .
$$

Integrating forward returns $\dot{\boldsymbol{s}}(t)$ and $\boldsymbol{s}(t) . \ddot{\boldsymbol{s}}(t), \dot{\boldsymbol{s}}(t)$ and $\boldsymbol{s}(t)$ are then sent to the adaptive-QP.

Finally, since the LMPCs are launched again and again and that it targets a zero velocity about $T \times N_{\text {steps }}$ seconds, the robot can never reach it completely so a stopping criterion is necessary. This criterion can simply be based on the current CoM velocity norm.

Discussion: in Eq. (6), we have split the equation of motion into a coupling part (first row) and a decoupled, block-diagonal part (next rows). As shown in [14], the coupling part corresponds to Newton and Euler equations. We enforce Newton's equation when combining LMPC results via Eq. (37), but chose to ignore Euler's equation (on the rate of change of the angular momentum) as a first approximation. This part will be further surveyed in future works. For now, we compute force polytopes by assuming that each limb is decoupled from each the others, and rely on the cost function of the FDQP to balance solutions and avoid adding unnecessary angular-momentum variations.

\section{Simulations}

The simulation sets up HRP-4 pushed against a wall, as depicted in Fig. 2. Here, it is assumed that the pre-impact phase (treated in [8]) is already done and only the postimpact phase remains to be controlled. The control loop of HRP-4 is $5 \mathrm{~ms}$, it is pushed on the back with a force of $300 \mathrm{~N}$ for $0.15 \mathrm{~s}$. The wall is $10^{\circ}$ inclined and we use an LMPC 1 with variables set as $N_{\text {steps }}=15$ and $T=15 \mathrm{~ms}$. The detection of the impact is done through the distance between the hands and the wall. As the algorithm computation time is higher than the control loop time, it launched is an estimated position of the hand touch the wall. This estimation is done with a simple Euler integration over $15 \mathrm{~ms}$ of the nearest point of a hand to the wall. To ensure that the adaptive-QP does not output high torque at the impact time, all tasks are removed but the minimization of the stiffness and damping coefficient. As soon as the LMPCs output the first results, a CoM trajectory task and posture task (with relatively low weight) is added. With an Intel(R) Core(TM) i7-4900MQ $\mathrm{CPU}$ at $2.8 \mathrm{GHz}$ (up to $3.8 \mathrm{GHz}$ ) with 4 cores and 8 running threads, the time required to compute the polytopes, the distribution force QP and the LMPCs is up to $15 \mathrm{~ms}$. To improve performance, the contact polytope $\mathrm{S}^{2}$ and the LMPCs are computed in parallel.

The stopping criterion has been set so that it is triggered when the norm of CoM velocity reached $1 \mathrm{~cm} \cdot \mathrm{s}^{-1}$. From

\footnotetext{
https://github.com/vsamy/Copra

${ }^{2}$ The polyhedral library (cddlib) itself is not multi-threadable.
}
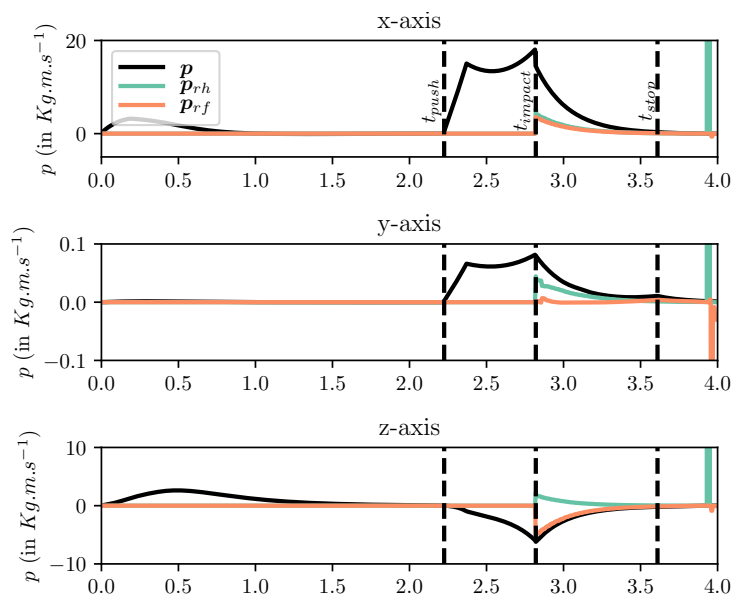

(a) Linear momentum and its splitted part

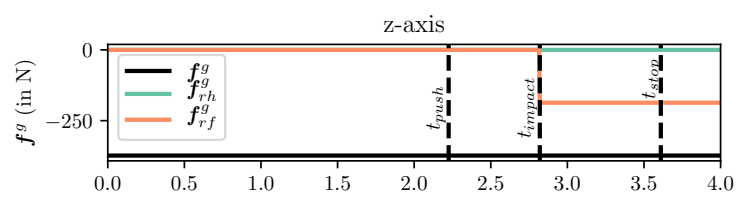

(b) Gravity force and its splitted part

Fig. 3: FDQP results through time.

there, the weight of the posture task is increased and the CoM trajectory is updated to a fixed set-point task at the current CoM position. We then stop the adaptive-QP and revert to the standard QP whole-body controller.

As the problem is symmetric the FDQP gives arms and legs the same amount of linear momentum and gravity hence Fig. 3a to Fig. 5 only display right side contacts/joints. The linear momentum of the CoM (Fig. 3a) smoothly decreases, on each axis, and reaches zero after roughtly $1.5 \mathrm{~s}$. Notwithstanding the symmetrical properties of the problem, we can denote a deviation of the linear momentum on the $y$ axis. In Fig. 3b the FDQP choses to compensate the $z$-axis of the gravity with only the foot which is coherent since the maximal forces of the feet are on this axis. It is also interesting to note that the FDQP may give one or multiple contacts the ability to go along with the whole-body linear momentum, rather than against it. This is quite visible on Fig. 3a on the $z$-axis where after the impact time, the sum of the two feet is greater than the robot linear momentum. Thus the feet alone have to compensate more than needed. This is due to the fact that the FDQP gives to the hand linear momentum a sign opposite to the whole-body one. So, LMPCs attached to the hands are seeing the robot going upward while the feet sees the robot going downward with 


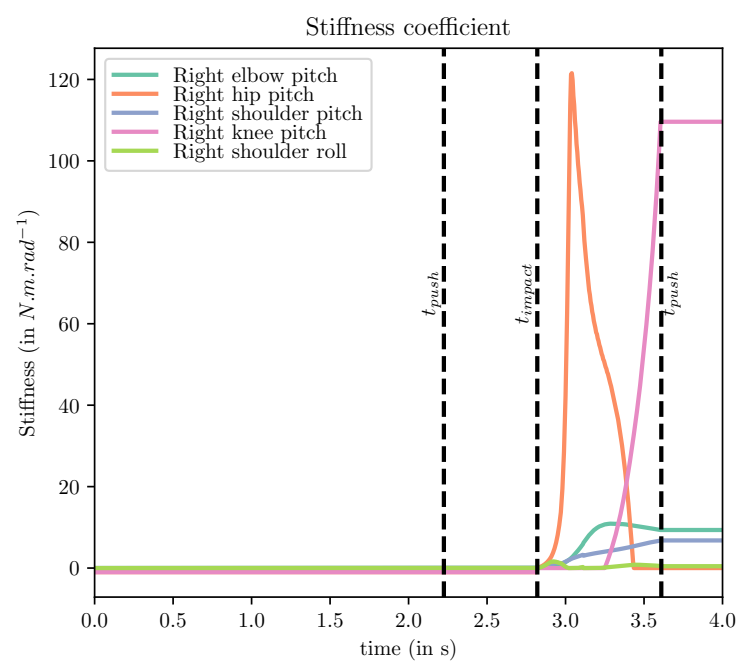

Fig. 4: Stiffness coefficient output of the adaptive-QP for several joints

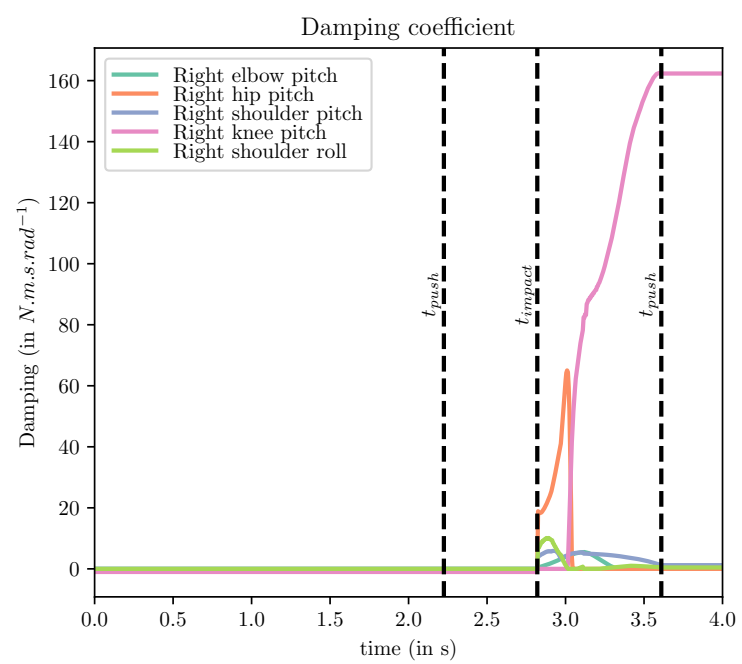

Fig. 5: Damping coefficient output of the adaptive-QP for several joints

higher velocity.

The adaptive-QP is able to find the stiffness (Fig. 4) and damping (Fig. 5) coefficient for arms and legs. At impact time, the damping coefficient dominates the stiffness one since the error term is almost zero whereas velocity is high. It is very clear that the hip joint is making most of the effort to fulfill the tasks. From common sense, the elbow joint should have particapated more since it is the one that can directly damp the linear momentum but it is not. This can be explained by the fact that the hip motor has a torque limit 3.5 times superior to the elbow's one. Around $t=3 \mathrm{~s}$, the knee joint takes over and is mainly set so that it maintains the height of the CoM and compensates gravity.

\section{CONClusion AND FUtURE WORK}

We have presented an approach that allows a humanoid robot to actively control its compliance after falling and impacting its environment with its arms and/or legs. Parallel model predictive controllers are run at each of the four contacting limbs, the joint action of which is to absorb the undesired linear momentum accumulated during fall. These predictive controllers are instantiated by a force distribution quadratic-program (FDQP) that optimally distributes the initial momentum and gravity force across contacting limbs. Despite relying on reduced dynamic models, all predictive controllers are constrained by contact polytopes that encode both friction and joint torque limitations.

The approach was validated in full-body simulations with a model of the HRP-4 humanoid robot in falling scenarios. The simulation showed corroborating results making the linear momentum vanish after few seconds, in an active compliance way.

With our previous work [7] [8], we have now a complete framework for fall handling from pre-impact posture adoption, to impact-time motor PD-gains automatic adaptation, and with the present work, post-impact active compliance with MPC to optimally absorb the impact and bring the robot to a safe rest.

\section{REFERENCES}

[1] C. G. Atkeson, B. P. W. Babu, N. Banerjee, D. Berenson, C. P. Bove, X. Cui, M. DeDonato, R. Du, S. Feng, P. Franklin, M. Gennert, J. P. Graff, P. He, A. Jaeger, J. Kim, K. Knoedler, L. Li, C. Liu, X. Long, T. Padir, F. Polido, G. G. Tighe, and X. Xinjilefu, "No falls, no resets: Reliable humanoid behavior in the darpa robotics challenge," in IEEERAS Int. Conf. on Humanoids, Nov 2015, pp. 623-630.

[2] J. Pratt, J. Carff, S. Drakunov, and A. Goswami, "Capture point: A step toward humanoid push recovery," in IEEE-RAS Int. Conf. on Humanoid Robots, 2006, pp. 200-207.

[3] A. Sherikov, D. Dimitrov, and P. B. Wieber, "Balancing a humanoid robot with a prioritized contact force distribution," in 2015 IEEE-RAS 15th International Conference on Humanoid Robots (Humanoids), Nov 2015, pp. 223-228.

[4] M. Battaglia, L. Blanchet, A. Kheddar, S. Kajita, and K. Yokoi, "Combining haptic sensing with safe physical interaction," in IEEE/RSJ Int. Conf. on Intelligent Robotics and Systems, Saint Louis, MO, USA, 11-15 October 2009, pp. 231-236.

[5] G. De Magistris, A. Pajon, S. Miossec, and A. Kheddar, "Optimized humanoid walking with soft soles," Robotics and Autonomous Systems, vol. 95, pp. 52-63, 092017.

[6] E. M. Hoffman, N. Perrin, N. G. Tsagarakis, and D. G. Caldwell, "Upper limb compliant strategy exploiting external physical constraints for humanoid fall avoidance," in 2013 13th IEEE-RAS International Conference on Humanoid Robots (Humanoids), Oct 2013, pp. 397402 .

[7] V. Samy and A. Kheddar, "Falls control using posture reshaping and active compliance," in IEEE-RAS Int. Conf. on Humanoids, 2015, pp. 908-913.

[8] V. Samy, K. Bouyarmane, and A. Kheddar, "Qp-based adaptive-gains compliance control in humanoid falls," in 2017 IEEE International Conference on Robotics and Automation (ICRA), May 2017, pp. $4762-$ 4767.

[9] K. Bouyarmane and A. Kheddar, "On weight-prioritized multitask control of humanoid robots," IEEE Transactions on Automatic Control, 2017.

[10] S. Caron, Q. C. Pham, and Y. Nakamura, "Zmp support areas for multicontact mobility under frictional constraints," IEEE Transactions on Robotics, vol. 33, no. 1, pp. 67-80, Feb 2017.

[11] K. Fukuda and A. Prodon, Double description method revisited. Berlin, Heidelberg: Springer Berlin Heidelberg, 1996, pp. 91-111.

[12] K. Bouyarmane and A. Kheddar, "On the dynamics modeling of freefloating-base articulated mechanisms and applications to humanoid whole-body dynamics and control," in 2012 12th IEEE-RAS International Conference on Humanoid Robots (Humanoids 2012), Nov 2012, pp. 36-42.

[13] H. Audren, J. Vaillant, A. Kheddar, A. Escande, K. Kaneko, and E. Yoshida, "Model preview control in multi-contact motionapplication to a humanoid robot," in 2014 IEEE/RSJ International Conference on Intelligent Robots and Systems, Sept 2014, pp. 40304035

[14] P.-B. Wieber, Holonomy and Nonholonomy in the Dynamics of Articulated Motion. Berlin, Heidelberg: Springer Berlin Heidelberg, 2006, pp. $411-425$. 